\title{
Le « réseautage » chez les entrepreneurs néo-ruraux
}

Par

Séverine SALEILLE

Docteur en Sciences de Gestion de l'ERFI-Université Montpellier 1

ATER

IUT de Sceaux, Université Paris Sud-11

PESOR

\section{Résumé}

Le cas des entrepreneurs néo-ruraux constitue un angle d'entrée nouveau dans la problématique du réseautage en entrepreneuriat car ils ne créent pas là où ils vivent mais au contraire dans un contexte géographique et bien souvent professionnel nouveau.

Une étude exploratoire a donc été conduite auprès de 20 cas implantés dans les Monts et Montagnes d'Ardèche afin de comprendre quelles pratiques de réseautage étaient mises en place.

Les résultats permettent de proposer une typologie des pratiques de réseautage. Ces dernières sont positionnées sur un plan à deux axes : la familiarité de l'entrepreneur avec le contexte fonctionnel de son entreprise et le contexte géographique dominant. Or, pratiques de réseautage local et non-local sont différentes.

\section{Introduction}

« Le carnet d'adresses du dirigeant et la qualité de son réseau relationnel comptent parmi les plus cités dans la liste des conditions de la réussite en création d'entreprise » (Verstraete et Saporta, 2006, p.310). D'ailleurs, dès les années 80 , suite à la thèse de l'encastrement de l'économique dans le social (Granovetter, 1985), aux travaux de la sociologie des réseaux sociaux et à la formulation de la notion de capital social (Bourdieu, 1980), s'est développé un courant de recherche voyant l'entrepreneur non plus comme solitaire ou fruit d'une détermination culturelle mais comme «encastré ». «Comme alternative à des modèles sous-socialisés ou sur-socialisés de l'entrepreneuriat, nous proposons une perspective qui voit l'entrepreneuriat comme encastré dans des réseaux de relations sociales continues ${ }^{1} \gg$ (Aldrich et Zimmer, 1985, p.8).

En reprenant la terminologie du sociologue Mitchell (1973), Szarka (1990) définit trois types de réseaux de relations sociales dans lesquels l'entrepreneur est encastré : le réseau personnel (qui comprend les contacts avec la famille, les amis et les connaissances), le réseau commercial (qui comprend les organisations avec lesquelles l'entrepreneur effectue des transactions commerciales) et le réseau de communication (qui comprend les organisations et individus par lesquels l'entrepreneur obtient des informations concernant son activité : consultants, institutions locales, etc.). Reprenant une classification de ce type (réseau personnel, réseau d'affaire, réseau informationnel), Julien et Lachance (2006) identifient un quatrième type de réseau : le réseau social (qui facilite l'insertion de l'entreprise dans son milieu). Ce dernier est à rapprocher de la notion de réseau symbolique utilisée par Johannisson (1987) pour désigner les liens communautaires par opposition au réseau personnel (constitué de relations d'amitiés) et au réseau de production (constitué des transactions avec des organisations sur le marché).Compte tenu de la multiplexité des liens (un même lien peut être à la fois personnel et professionnel) et des échanges (un même lien peut permettre une transaction économique, être source d'informations et faciliter l'insertion dans le milieu), nous utiliserons par la suite le terme "réseau personnel ». Ce terme est utilisé comme synonyme de « réseau égocentré », c'est à dire pour désigner non pas simplement les alter ${ }^{2}$ (membres du réseau) avec lesquels ego (l'entrepreneur) entretient des relations de nature privée mais l'ensemble des alter (individus ou organisations) avec lesquels ego est en relation directe et qui ont, comme le propose Hansen (1995) au travers de la notion de réseau « actif », joué un rôle dans la création de l'entreprise, quelle que soit la nature de la relation (privée ou non). 
Les revues de littérature de Johannisson et Monsted (1997), O’Donnell et al. (2001), Hoang et Antoncic (2003) et Drakopoulou Dodd et al. (2006) montrent que la plupart des travaux dans le courant de l'entrepreneur «encastré $»^{3}$ ont porté sur les effets du réseau personnel et la configuration de ce dernier, l'objectif étant d'identifier une configuration en quelque sorte optimale. Concernant la constitution et la mobilisation du réseau personnel, les travaux sont moins nombreux. Or, la pratique de gestion que nous qualifions de réseautage est essentielle lors de la création d'une entreprise. "Mobiliser des ressources pour poursuivre des opportunités requiert des contacts, des connaissances et de la confiance. Mobiliser des ressources implique aussi de demander à d'autres de fournir des moyens financiers et des efforts pour une entreprise dont l'avenir est incertain. L'entrepreneuriat est donc une activité essentiellement de mise en réseau. » (2006).

Pour Reynolds (Dubini et Aldrich, 1991, p.306), « la preuve principale de l'importance des réseaux sociaux est que la plupart des entrepreneurs créent leur entreprise «à la maison "; c'est à dire dans un contexte géographique - même communauté et même juridiction politique - comme industriel familier. $\gg^{6}$ (p. 64). Cela leur permet d'avoir de multiples contacts pour trouver des fonds, des fournisseurs, des clients, etc. Cela diminue aussi le risque lié à la création. Ainsi, en créant dans des contextes familiers, l'entrepreneur peut obtenir le maximum d'informations utiles mais, surtout, ne prend pas le risque que certains profitent de sa vulnérabilité. En effet, l'encastrement dans un contexte familier fait que les relations économiques sont également sociales. Or, les raisons et intérêts du recours par les entrepreneurs aux relations sociales plutôt que de marché ont été montrés (Johannisson, 1986; Starr et MacMillan, 1990) : obtention de ressources à un coût moindre, voire obtention de ressources qui n'auraient pas pu l'être, même à un coût supérieur, dans le cadre d'une relation marchande. De plus, des comportements de confiance et d'absence d'opportunisme sont attendus. Les avantages de créer « à la maison » (ce terme devant être compris comme « dans un environnement géographique et professionnel familier ») sont, donc, bien réels.

Or, l'entrepreneuriat néo-rural va à l'encontre de cette propension des entrepreneurs à créer là où ils vivent. En effet, de plus en plus de personnes décident de quitter la ville pour s'installer à la campagne et y créer une entreprise. Le repeuplement des campagnes qui s'est amorcé dans les années 70, en France comme dans la plupart des pays développés, s'est accrû et transformé ces dernières années. Selon l'Observatoire des Territoires (1991), 1,8 millions de personnes, dont plus de 800000 actifs, ont quitté un pôle urbain pour la campagne entre 1990 et 1999. Et cette tendance, qui touche, selon les résultats des enquêtes annuelles de recensement de 2004 et 2005, de plus en plus, les espaces ruraux les plus isolés (2005) n'est, semble-t-il, pas prête de s'inverser. Ainsi, selon un récent sondage ${ }^{7}$, plus de 8 millions de citadins (39\%) désireraient s'installer à la campagne et pas nécessairement pour y passer leur retraite. En effet, $50 \%$ des citadins souhaitant vivre à la campagne veulent le faire en tant qu'actifs et $23 \%$ envisagent même de changer d'employeur ou d'activité. Une bonne partie d'entre eux sera amenée à créer son entreprise pour réaliser ce souhait de vie à la campagne.

Par conséquent, le cas des entrepreneurs néo-ruraux constitue un angle d'entrée nouveau, et particulièrement intéressant, dans la problématique du réseautage. En effet, comment ces entrepreneurs néo-ruraux, qui ne créent pas « à la maison » mais, au contraire, dans un contexte géographique et parfois professionnel nouveau, parviennent-il à se constituer le réseau personnel si indispensable à la création et au développement de leur activité ?

Dans une première partie, nous montrerons en quoi le cas des entrepreneurs néo-ruraux peut constituer un angle d'entrée nouveau dans la problématique du réseautage en entrepreneuriat. Dans une seconde partie, nous présenterons les résultats d'une étude exploratoire sur les pratiques de réseautage des entrepreneurs néo-ruraux. 


\section{Le cas des entrepreneurs néo-ruraux : un angle d'entrée nouveau dans la problématique du réseautage}

Après avoir présenté comment le réseautage est traité dans la littérature entrepreneuriale, nous montrerons en quoi l'entrepreneur néo-rural, de part ses particularités, peut présenter des comportements particuliers en termes de réseautage.

\subsection{La problématique du réseautage en entrepreneuriat}

Dans cette section, nous montrons que le réseautage peut être défini comme la constitution et la mobilisation du réseau personnel et présentons comment chacun de ces deux aspects est traité dans la littérature.

\subsubsection{Le réseautage : constitution et mobilisation du réseau personnel}

Le réseautage peut être conçu comme, seulement, une activité de mobilisation du réseau personnel (Aldrich et al., 1987; Hill et al., 1999) ou comme une activité à la fois de constitution et de mobilisation du réseau personnel (Carson et al., 1995; Morel et Redor, 2006). La définition de Jack (2005) nous semble la plus adaptée car elle distingue bien les deux processus (constitution et mobilisation) : "Un processus qui se développe dans le temps et qui implique, pour l'entrepreneur, d'activer ses contacts latents pour manifester des liens et transférer la relation à une situation entrepreneuriale, d'identifier un besoin entrepreneurial et de localiser l'individu dans le réseau qui peut l'aider sur le besoin en question. $»^{8}$.

Le réseautage de l'entrepreneur a un effet important sur les performances de celui-ci. Ainsi, l'étude de Chell et Baines (2000) montre que les entrepreneurs qui sont les plus « réseauteurs » ont des niveaux de performance supérieurs à ceux qui le sont moins ou pas du tout. Cependant, un niveau d'activité « réseau » important n'implique pas nécessairement des performances supérieures. Ainsi, les résultats de l'étude empirique de Watson (2006) montrent que la relation entre performance et activité « réseau » de l'entrepreneur suit une courbe en U inversé. Il existe un effet de seuil au-delà duquel l'activité « réseau » de l'entrepreneur est excessive et contre-productive. Réseauter, constituer et mobiliser un réseau personnel, nécessite certaines compétences sociales. Baron et Markman (2000, 2003) identifient celles qui sont les plus utiles dans l'activité entrepreneuriale : perception sociale, management de la première impression, persuasion et influence, adaptabilité sociale, expressivité. Selon Vissa et Anand (2006), le style de réseautage des entrepreneurs dépend de trois compétences : leur capacité à mémoriser des informations personnelles et contextuelles au sujet des alter, leur capacité à entrer en contact avec des alter « prestigieux » dans le contexte de la création et, enfin, leur capacité à mettre en relation des alter. Ces capacités dépendraient de l'expérience professionnelle et de la personnalité de l'entrepreneur. Lux et Ferris (2006) développent, également, un modèle théorique montrant en quoi les entrepreneurs qui disposent de compétences sociales ont un avantage compétitif dans le développement d'un réseau personnel : cela leur permet d'identifier les personnes potentiellement utiles dans leur réseau social actuel, ils sont capables de développer des relations efficaces avec un grand nombre de contacts potentiels, et ce avec moins d'effort.

\subsubsection{L'importance du réseau préexistant de l'entrepreneur dans la constitution du réseau personnel}

La constitution du réseau personnel a fait l'objet de peu de travaux. Pour les sociologues Minguet et Moreau (2006), deux explications peuvent être apportées au fait que la formation du réseau constitue un « impensé ». En premier lieu, beaucoup d'auteurs considèrent le réseau personnel comme une variable indépendante de l'entrepreneur : soit le réseau est préexistant (institutions d'accompagnement présentes sur le territoire d'implantation, par exemple), soit l'entrepreneur dispose de réseaux « naturels », qui lui 
sont donnés à la naissance, et sur lesquels il n'a pas de prise (degré de cohésion de sa famille ou de sa communauté d'origine, par exemple). En second lieu, d'autres auteurs considèrent que l'initiation de l'échange se fait sur la base d'un utilitarisme économique : la confiance entre les acteurs est une condition mineure de l'échange et, en fait, le réseau personnel ne se forme que si l'intérêt à échanger est optimum.

Les travaux portant sur l'évolution du réseau personnel soulignent que, pendant la phase de constitution du réseau, le rôle du réseau préexistant de l'entrepreneur est crucial. Le modèle de Butler et Hansen (1991) identifie 3 phases dans l'évolution du réseau personnel. La première phase comprend la période avant la création de l'entreprise. Le réseau social du créateur y joue un rôle très important en fournissant des informations et ressources utiles à l'identification de l'opportunité. Pendant cette phase, commence à apparaître un réseau plus professionnel qui comprend les individus et organisations en mesure de répondre aux besoins immédiats de l'entreprise en création. Cela ne signifie pas, pour autant, que le réseau social ne joue plus de rôle : il continue à fournir des informations utiles à l'identification de nouvelles opportunités et il constitue une sorte de «stock» de relations dans lequel l'entrepreneur va pouvoir puiser pour former le réseau professionnel. Ainsi, durant la deuxième phase, la phase de démarrage de l'entreprise, le réseau professionnel est en fait hybride : il comprend à la fois des individus issus du réseau social préexistant et de nouveaux individus et organisations avec qui l'entrepreneur entretient des liens professionnels (fournisseurs, clients, apporteurs de capitaux, etc.). Alors que, dans la troisième phase, l'entreprise commence à faire des profits et envisage la croissance, ce réseau professionnel évolue vers un réseau stratégique, les liens avec certains acteurs du réseau professionnel pouvant évoluer vers le partage d'actifs (comme des connaissances techniques ou la réputation).

Les quelques travaux empiriques sur ce thème soulignent, également, que la constitution du réseau personnel se fait essentiellement par activation d'un réseau préexistant (social et/ou professionnel). Ainsi, à partir de l'étude de huit cas d'entreprises nouvellement créées (Etats-Unis, secteur informatique), Hite (2005) identifie trois modes d'entrée dans le réseau : relation personnelle, relation économique et mise en relation par un tiers. A chacun, de ces modes d'entrée correspond un type de confiance « a priori »: confiance en la bienveillance de l'autre, confiance en la compétence de l'autre et confiance en la qualité de l'information fournie par la tierce personne. Sur les 66 liens forts étudiés, l'auteur trouve que $66 \%$ étaient des relations personnelles, $14 \%$ étaient des relations économiques et $20 \%$ sont entrés en relation avec ego par le biais d'un tiers. Bien que les liens faibles ne soient pas étudiés, cette étude confirme le poids du réseau préexistant dans la constitution du réseau personnel puisque la quasi-majorité des liens forts sont issus directement ou indirectement (par cooptation) de ce dernier. Dans la perspective de mieux comprendre le processus de formation d'un lien nouveau, Anderson et Jack (2002) tentent de définir les « règles » de création d'un lien à partir d'entretiens auprès d'entrepreneurs et d'une étude expérimentale (mise en relation de deux entrepreneurs). Les étapes vont de la rencontre et l'exploration d'affinités et de points communs jusqu'à l'identification de complémentarités mutuelles et la découverte de potentiels de collaborations, l'étape ultime étant le partage de capital social.

\subsubsection{La mobilisation du réseau personnel : entre réseautage instrumental et affectif}

Deux formes de réseautage peuvent être distinguées : l'un est instrumental et l'autre est affectif.

Si l'entrepreneur s'appuie au départ essentiellement sur son réseau social, donc sur un réseautage affectif, il a intérêt, par la suite, à chercher de l'aide et des informations au-delà de ce réseau social. Se développe alors un réseautage plus instrumental. Ainsi, l'étude de Hill et al.(1999) montre comment le réseau personnel orienté " marketing » évolue tout au long de la vie de l'entreprise (il s'agit d'entreprises orientées croissance). Au début, le réseau est avant tout social, centré autour de la famille et des amis. Cependant, ces contacts n'ont pas nécessairement les savoirs-faire et expertises nécessaires. En effet, leur rôle est surtout un rôle de soutien face aux risques que court l'entrepreneur. Les auteurs montrent que l'entrepreneur a intérêt à chercher de l'aide et des informations au-delà de ce réseau social. Ces relations professionnelles, des liens faibles, deviennent de plus en plus importantes au fil de la 
réussite de l'entreprise. En particulier, le rôle des banques et des fournisseurs grandit. En revanche, les clients, les employés ou les structures d'accompagnement locales ne semblent pas jouer de rôle clef dans le réseau personnel des entreprises étudiées

S'appuyant sur 3 cas d'entrepreneurs, deux cas de " réussite » à un cas d' " échec ", l'étude de Drakopoulou Dodd et al. (2006) montre que si sur le court-terme, le réseautage instrumental peut apporter divers bénéfices, il est nécessairement amené à se transformer au fil du temps en réseautage affectif. L'étude montre que les moyens d'entretien du réseau sont la réciprocité de l'échange et le partage de valeurs communes. «Ces normes et représentations partagées sont utilisées comme un critère d'embauche des employés, mais aussi comme un moyen de valider le choix de partenaires extérieurs. C'est également vu comme une base pour développer des relations amicales, pour développer des liens forts à partir de liens faibles. $»^{9}(\mathrm{p}$. 135). Le passage d'un réseautage instrumental à un réseautage affectif nécessite l'acquisition des trois formes de confiance évoquées par Hite (2005) : confiance en la bienveillance, confiance en la compétence et confiance sociale. L'étude de Bowey et Easton (2007) montre qu'il existe un modus operandi pour créer ou détruire la confiance présente dans une relation. Les activités facilitant la création de confiance sont l'échange de services, les relations sociales (dîner, activités sportives, etc.), la résolution de problèmes en commun, la réalisation des tâches répondant aux attentes et l'utilisation de communications transparentes.

D'après la littérature, le réseautage de l'entrepreneur est donc présenté comme étant, au départ, affectif (il s'appuie essentiellement sur son réseau préexistant pour constituer son réseau personnel). Au cours du démarrage de l'entreprise, il devient plus instrumental. Mais à long terme, ce réseautage instrumental est amené à se transformer en réseautage affectif. Dès lors, en quoi l'entrepreneur néo-rural, de part ses particularités, peut impliquer une forme de réseautage particulier?

\subsection{Les particularités de l'entrepreneur néo-rural : quid en termes de réseautage?}

Nous adhérons à l'idée d'une intra-singularité de l'entrepreneuriat (Levy-Tadjine et Paturel, 2006). Ainsi, de nombreux travaux s'intéressent à des types d'entrepreneurs singuliers et en identifient les particularités: chercheurs-créateurs (Emin, 2003), entrepreneurs immigrés (Levy-Tadjine, 2004), éco-entrepreneurs (Berger-Douce, 2006), cyber-entrepreneurs (Carrier et al., 2002), entrepreneurs de l'économie sociale et solidaire (Boncler et Hlady-Rispal, 2002), etc. De la même façon, notre analyse du phénomène de l'entrepreneuriat néo-rural nous a conduit à relever trois particularités fortes chez ces entrepreneurs : il s'agit de migrants qui s'implantent en milieu rural avec des motivations plus existentielles qu'économiques.

\subsubsection{Un entrepreneur migrant...}

La première particularité des entrepreneurs néo-ruraux est l'imbrication de la migration et de la création. Cela implique que ces entrepreneurs ne peuvent bénéficier des intérêts, liés à l'insertion du créateur dans un réseau social, de la création «à la maison » au sens de Reynolds (1991) Cet aspect a fait l'objet de peu de travaux académiques. En revanche, c'est une préoccupation majeure des structures d'accompagnement. Ainsi, le Collectif Ville-Campagne (2003) distingue trois temps forts dans le parcours d'un candidat à l'installation en milieu rural : le temps de l'idée, le temps du projet (qui inclut le projet de vie, le projet professionnel, mais aussi la recherche d'un territoire d'implantation) et le temps de l'installation (où l'intégration dans le milieu d'accueil est particulièrement importante).

De plus, la migration constitue un événement perturbateur du réseau social d'un individu. "Les moments de recomposition peuvent être liés à des changements de cycle de vie (décohabitation, insertion professionnelle, retraite), à des ruptures professionnelles (changement d'emploi, licenciement), familiales (décès, divorce), à des événements contingents, ou encore à des mobilités géographiques. Ce sont évidemment les mobilités géographiques qui ont le plus d'impact sur la composition spatiale des 
réseaux » (Grossetti, 2001, p.216). Des travaux sur l'impact de la migration sur le réseau social d'un individu (Assogba et al., 2000; Bidart et Fribourg, 2004), montrent que le déménagement dans un espace d'accueil différent de son espace d'origine implique une recomposition du réseau social avec le maintien de liens forts dans le milieu d'origine et la création de liens nouveaux, en général faibles au départ, dans le milieu d'accueil.

\subsection{2... qui s'implante en milieu rural...}

La deuxième particularité est la localisation rurale. La littérature montre que cette localisation, de part ses caractéristiques géo-démographiques (éloignement, manques en ressources, faible densité) et socio-culturelles (interconnaissance, appartenance à une communauté, solidarités), implique des pratiques de gestion spécifiques : stratégie coopérative implicite, importance du bouche à oreille dans la communication, imbrication des réseaux sociaux et professionnels (Shields, 2005).

En particulier, une controverse existe sur les pratiques de réseautage à adopter dans un tel milieu (Joyal, 2000). Faut-il profiter de l'interconnaissance qui y règne pour s'y créer un réseau dont on peut penser qu'il sera particulièrement coopératif? Au contraire, faut-il aller chercher dans d'autres milieux les ressources que l'environnement immédiat ne peut fournir faute d'un tissu social suffisamment dense ?

\subsection{3.....avec des motivations plus existentielles qu'économiques}

La troisième particularité est la nature des mobiles de ces entrepreneurs. La création de l'entreprise s'inscrit dans un projet de vie plus large, un projet (souvent familial ou de couple) d'amélioration de la qualité de vie par l'installation à la campagne.

Une des formes de qualité de vie à la campagne est le fait de reconnaître facilement et d'être facilement reconnu (Font, 2000). Donc, une des motivations à la migration peut être de rompre avec l'anonymat des grandes villes. Si la création de l'entreprise s'inscrit dans cette optique, cela peut impliquer des pratiques de réseautage particulières.

La problématique du réseautage est donc une problématique majeure pour l'entrepreneur néo-rural : il s'installe dans un milieu où le réseau compte beaucoup (interconnaissance) alors que son réseau social est en pleine recomposition et qu'il souhaite retrouver un type de relations plus humaines. Par conséquent, nous avons conduit une étude exploratoire sur les pratiques de réseautage mises en place par ce type particulier d'entrepreneur qu'est l'entrepreneur néo-rural.

\section{Les pratiques de réseautage des entrepreneurs néo-ruraux : une étude exploratoire}

Une étude qualitative et interprétative a été conduite auprès de 20 nouveaux arrivants créateurs d'entreprises dans les Monts et Montagne d'Ardèche. Celle-ci nous permet de proposer une typologie de pratiques de réseautage chez les entrepreneurs distinguant pratiques de réseautage local (constitution et entretien/développement du réseau personnel local) et pratiques de réseautage non local (constitution et entretien/développement du réseau personnel non local).

\subsection{Méthodologie et terrain d'étude}

S'inscrivant dans le courant interprétatif, la démarche retenue est qualitative et exploratoire. L'étude porte sur 20 cas de nouveaux arrivants ayant créé une entreprise dans les Monts et Montagne d'Ardèche. Malgré ses handicaps (zone de moyenne montagne, de faible densité, mal desservie et éloignée des principales villes du sud-est de la France), ce territoire attire, en effet, de nombreux nouveaux arrivants 
(70\% des communes y ont un solde migratoire positif) et s'est doté d'une politique d'accueil de nouveaux habitants et de nouvelles activités (dans le cadre d'un Groupe d'Action Locale du programme européen Leader+).

Les enquêtés ont été identifiés par le bais d'informateurs clefs (structures d'accompagnement intervenant sur le territoire), l'exploitation de fichiers (en particulier le fichier SIRENE) et le recours à l'effet «boule de neige » (quand les enquêtés deviennent eux-même informateurs-clefs). En ce qui concerne les caractéristiques socio-démographiques des enquêtés, notre échantillon est composé surtout de personnes vivant en couple (16), dont une majorité a des enfants (9). Il comprend aussi bien des créateurs au début ( 7 ont entre 20 et 35 ans), au milieu ( 6 ont entre 35 et 45 ans) ou en fin ( 7 ont entre 45 et 55 ans) de vie professionnelle. La majorité des migrations ont eu lieu entre 2001 et 2004. 8 enquêtés viennent du Sud-Est de la France (Lyon, Grenoble, Annecy, Marseille, etc.). Pour les 12 autres, la migration est de plus grande ampleur : 9 viennent du reste de la France (région parisienne surtout) et 3 de l'étranger. Les caractéristiques des entreprises créées sont présentées dans le tableau 1.

Tableau 1 : Caractéristiques des 20 cas (date de création, statut juridique et activités)

\begin{tabular}{|l|l|l|l|}
\hline Cas & Création & Statut & Activités \\
\hline E1 & déc-03 & SARL & Création de sites Internet, affiliation \\
\hline E2 & févr-02 & SARL & Hébergement touristique, restaurant, espace de relaxation \\
\hline E3 & sept-02 & SE ${ }^{10}+$ EI & Formations-conseils, activité agritouristique \\
\hline E4 & août-03 & EI & Vente de livres, CD et DVD \\
\hline E5 & févr-04 & SARL & Vente et réparation de véhicules, location de Quads \\
\hline E6 & mai-02 & EI & Agence de traduction, chambre et table d'hôtes \\
\hline E7 & sept-03 & EI & Conseils en organisation \\
\hline E8 & févr-05 & EURL & Etude et fabrication de produits électroniques \\
\hline E9 & Mars-04 & SARL & Création de Sites Internet et supports publicitaires \\
\hline E10 & janv-05 & EI & Traduction \\
\hline E11 & nov-03 & SARL & Conseils en systèmes d'information \\
\hline E12 & sept-03 & EI & Conseils en gestion de patrimoine et agence immobilière \\
\hline E13 & juil-05 & EI & Vente de produits cosmétiques et de terroir, coaching sportif \\
\hline E14 & mars-05 & EI & Agence de communication \\
\hline E15 & avr-04 & EI & Vente de produits en bois \\
\hline E16 & oct-03 & SE & Formations-conseils \\
\hline E17 & avr-04 & EI & Accueil de personnes handicapées, chambre et table d'hôtes \\
\hline E18 & nov-04 & EI & Vente et fabrication de bijoux en bois précieux \\
\hline E19 & avr-03 & EURL & $\begin{array}{l}\text { Traduction, opérateur en télécommunication, projets fabrication d'objets } \\
\text { technologiques en bois et distribution de produits bio }\end{array}$ \\
\hline E20 & juin-03 & SARL & $\begin{array}{l}\text { Tournage sur bois artistique et à façon, restauration de meubles, organi- } \\
\text { sation de stages de tournage }\end{array}$ \\
\hline
\end{tabular}

Les données ont été recueillies par entretiens semi-directifs rétrospectifs. Pour 5 cas, nous avons, également, réalisé un second entretien 6 à 18 mois après le premier. Beaucoup moins formalisé que le premier, ce second entretien a permis surtout de recueillir des données manquantes, d'avoir un retour sur une première analyse des données et de recueillir des informations sur l'évolution de l'entreprise et du réseau. Ces entretiens ont été enregistrés puis retranscrits. Le guide d'entretien permettait à l'enquêté de retracer son parcours de créateur d'entreprise et d'évoquer les individus et organisations ayant joué un rôle durant ce parcours, la nature de ce rôle, le mode de rencontre, le type de lien et la façon dont il est 
activé. Après une phase introductive sur les raisons de la migration et de la création, l'entretien prenait donc la forme d'un récit de vie contextualisé à la création de l'entreprise structuré en trois grandes phases (la réflexion sur le projet, la préparation du projet et le démarrage de l'entreprise) avec des questions incitant à citer les alter étant intervenus et leur rôle. Ensuite, nous invitions l'enquêté à faire un bilan de la création et du réseau. Puis les relations au milieu d'accueil et les projets étaient évoqués.

Pour l'analyse des données, nous avons procédé à un codage de type bottom-up sous le logiciel d'analyse thématique NVivo7 à partir d'une pré-structuration faible des catégories. Il s'agissait donc de partir des données brutes pour aller vers des catégories plus conceptuelles, en regroupant les codes de première catégorie (au plus près du discours de l'enquêté) sous un code un plus abstrait et ainsi de suite. Une matrice d'analyse de réseau, par cas puis inter-cas, a également été constituée. Cette matrice indiquait pour chaque alter : le contexte social le liant à ego, la localisation géographique, le rôle joué, le stade d'intervention et, enfin, qui est à l'initiative de l'échange. Ses analyses ont abouti à une typologie des pratiques de réseautage, exposée dans les sections suivantes.

\subsection{Proposition d'une typologie de pratiques de réseautage}

Suite à l'analyse des transcriptions d'entretiens, nous proposons la typologie suivante. Celle-ci classe les diverses pratiques de réseautage (+ indiquant que cette pratique est très utilisée dans le contexte, - indiquant qu'elle est peu utilisée) sur un plan comprenant deux axes : la familiarité avec le contexte fonctionnel et le contexte géographique dominant.

Figure 1. Pratiques de réseautage en fonction de la familiarité avec le contexte fonctionnel et du contexte géographique dominant

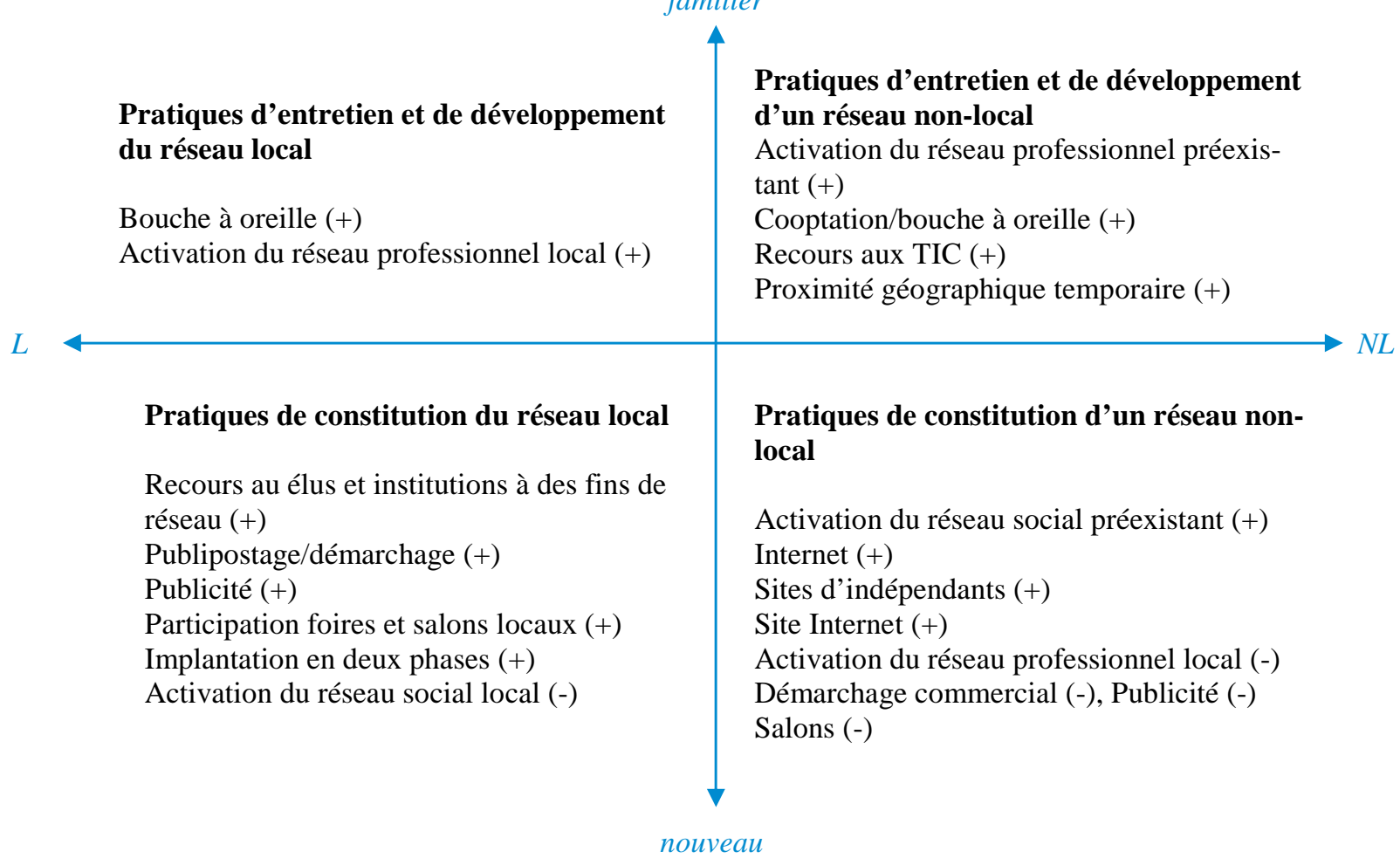

Le premier axe du plan est la familiarité avec le contexte fonctionnel. Par contexte fonctionnel, nous entendons le métier, le secteur d'activité, la clientèle de l'entreprise. Si la rupture géographique est une caractéristique récurrente chez les entrepreneurs néo-ruraux, la littérature est plus partagée en ce qui concerne la rupture professionnelle. Ainsi, dans une étude portant sur les nouveaux venus dans le sud 
des Cévennes dans les années 1970-1980, Catanzano (1987, cité par Chevalier, 2003) montre que l'impossibilité d'appliquer leur compétence première sur place les a conduit à modifier totalement leur situation professionnelle en exploitant leurs compétences secondes ou leurs passions. Cependant, Negro (1995) montre qu'en fait quand l'activité créée est une activité intellectuelle, c'est rarement le cas. Au contraire, ces créations correspondent plutôt à une forme d'essaimage. Nous retrouvons cette diversité de situations au sein de notre échantillon. Ainsi, pour 8 enquêtés, le contexte fonctionnel est plutôt nouveau : ils n'ont pas ou peu d'expérience du métier, ont rarement déjà créé et n'ont aucun réseau professionnel préexistant (sauf un enquêté, mais son réseau est associatif et se révélera peu « rentabilisable »). Au contraire, pour 8 autres enquêtés, le contexte fonctionnel est familier : tous ont une expérience du métier, du secteur et un réseau professionnel préexistant (sauf une enquêtée dont l'activité est en continuité avec son vécu extra-professionnel et non professionnel). La moitié ont même déjà créé une entreprise ou une activité secondaire. Enfin, 4 enquêtés ont créé deux activités totalement différentes: une pour laquelle le contexte fonctionnel est familier et une pour laquelle il est totalement nouveau, la première activité étant destinée à financer le lancement de la seconde.

Le deuxième axe du plan est le contexte géographique où se situe les alter mobilisés par l'entrepreneur. Il peut s'agir du milieu d'accueil (L pour local) ou d'autres milieux (NL pour non-local), parmi lesquels le milieu d'origine. En comparant entrepreneurs néo-ruraux et autochtones Anderson (2000) a montré que l'expérience d'un environnement différent (urbain) permet aux néo-ruraux de voir leur environnement local comme une opportunité alors que les autochtones le voient comme une contrainte. Ainsi, les néo-ruraux sont attirés par les propriétés esthétiques et physiques du milieu rural et y perçoivent des opportunités d'affaires. Les autochtones, eux, sont beaucoup plus focalisés sur le marché local et identifient des opportunités dans les besoins locaux. Pour Kalantaris et Bika (2006), la différence fondamentale entre néo-ruraux et autochtones ne réside pas dans la perception qu'ils ont de leur localisation rurale mais dans la manière dont ils s'encastrent dans leur environnement local et utilisent les ressources locales. Les néo-ruraux utilisent beaucoup moins les ressources locales que les autochtones, en particulier pour leurs approvisionnements, leurs ventes et les sources d'informations sur les opportunités de marché. Nos résultats montrent que s'il existe, chez les néo-ruraux, une grande diversité de comportements en termes d'ancrage local, un élément est toujours présent : ils s'entourent à la fois d'un réseau personnel local et d'un réseau personnel non-local. Or, que ce soit pour la constitution, l'entretien ou le développement d'une relation, les pratiques différent grandement selon que l'alter se situe dans le milieu d'accueil ou est plus éloigné. Nous exposons et discutons les pratiques de réseautage local, puis celles de réseautage non-local dans les deux sections suivantes.

\subsection{Le réseautage local : d'abord instrumental puis affectif}

Parce qu'il est nouvel arrivant et que son réseau préexistant sur place est petit et peu adapté, la constitution d'un réseau personnel dans le milieu d'accueil (quadrant en bas à gauche de la typologie) implique pour l'entrepreneur l'utilisation de pratiques formelles, et donc un réseautage plutôt instrumental. Ce n'est que par la suite, quand un premier noyau de réseau local est constitué et donc quand le contexte fonctionnel local devient plus familier (quadrant en haut à gauche de la typologie) que le réseautage peut devenir plus affectif.

\subsubsection{Les insuffisances du réseautage affectif pour la constitution du réseau personnel local}

Le réseautage affectif, c'est à dire s'appuyant essentiellement sur le réseau préexistant, s'avère être insuffisant pour la constitution du réseau personnel local.

Etant nouvel arrivant, l'entrepreneur néo-rural a, au moment de la création, un réseau préexistant sur place peu développé. Le réseautage a donc un double objectif : intégration personnelle et intégration de l'entreprise. C'est par exemple ce que souligne cette créatrice d'une boutique de vente de produits cosmétiques et de terroirs, ainsi que d'une activité de coaching sportif et massages : "C'est un 
bouleversement total, c'est un gros changement, il y a une grosse part d'adaptation. C'est beaucoup de tracas, beaucoup de soucis dans le sens où il faut à la fois tout gérer, il faut gérer à la fois l'adaptation locale, à la fois le développement d'une entreprise, la vie personnelle, la vie familiale en plus. Les travaux se rajoutent. » (E13). Or, de façon conforme à ce qu'Assobga et al. (2000) évoquent pour les migrations de jeunes vers les villes, les enquêtés ont, souvent, tendance à se constituer un réseau social composé essentiellement d'autres néo-ruraux. Cela peut constituer un handicap pour la constitution d'un réseau local professionnel pertinent. Par exemple, ce créateur de sites Internet a, avant la création, suivi une formation à la création d'entreprise qui lui a permis de nouer de nombreux contacts sur place, essentiellement avec d'autres néo-ruraux. Il en tire la conclusion suivante : "Pour un néo, se constituer un réseau que d'autres néos est un inconvénient au niveau professionnel mais au niveau émotionnel, il est possible de créer un réseau affectif très fort. »(E1).

Cette tendance à s'entourer surtout de néo-ruraux peut s'expliquer par le fait que les enquêtés ressentent une certaine fermeture des autochtones vis à vis d'eux. C'est, par exemple, ce que souligne ce tourneur sur bois quand des conseils aux nouveaux arrivants lui sont demandés : " Le plus dur c'est d'être un étranger, de venir d'autre part que de l'Ardèche ((rires)), c'est encore plus difficile. Ça risque de poser de gros problèmes. L'acceptation n'est pas vraiment facile. [...] Les gens veulent bien voir les jeunes arriver, s'implanter mais pas trop. »(E20). Cette fermeture se traduit par l'existence d'un délai d'acceptation par le milieu local. Ceci rejoint la proposition de Schields (2005) qui donnait cet argument pour expliquer que les néo-ruraux avaient plus de difficultés à accéder à un marché local que les autochtones. D'ailleurs ce même tourneur sur bois évoque ce délai : "Nous, on a été regardés à la loupe parce qu'on restera, on est des parisiens et on restera des parisiens toute notre vie. Et puis, à un moment donné, les gens regardent. Ils sont encore là ? Un an, bon, c'est bien. Deux ans, ils sont encore là ? Oui, donc ils doivent faire quelque chose. On va pousser la porte. Là, en ce moment, on a le retour de gens qui viennent et qui disent : voilà, on a vu, on passe devant tous les jours et on a vu que vous faisiez des choses. Alors que c'est des gens qui passent devant tous les jours, savent très bien ce qu'ont fait et ont attendu deux ans avant de rentrer dans la boutique. »(E20).

Compte-tenu de ces freins, l'activation du réseau préexistant local n'est pas une pratique suffisante pour se constituer un réseau personnel local. Cependant, parce qu'ils constituent une première base - même embryonnaire - de réseau préexistant local, le rôle des référents locaux est particulièrement important. La majorité des enquêtés (16) avait effectivement déjà des liens (amis, famille, connaissance) en Ardèche avant de s'y implanter. Cela rejoint les conclusions de l'étude de Roussel et Bégon (2005) qui montrait que les néo-ruraux n'étaient finalement pas si nouveaux que ça, la plupart ayant au moins un référent sur place ou étant déjà rentrés en contact avec le territoire lors de vacances. Par exemple, cette traductrice et propriétaire de chambres d'hôtes a constitué une bonne partie de son réseau local à partir d'un seul contact sur place : la propriétaire d'un hôtel. «Le réseau qui a été très utile a été à partir de l'hôtel X ((propriétaire connue avant l'installation)) et après l'agent immobilier. Et l'agent immobilier qui m'a menée à la banque, la banque et l'agent immobilier qui m'ont envoyée chez le notaire, le notaire qui m'a envoyée chez mon comptable. »(E6). De même, ce formateur a constitué la majorité de son réseau local a partir d'une amie dirigeant une association sur place : «L'association $X$, ça a été un peu la plaque tournante de tout ce que $\mathrm{j}$ ' ai pu tisser comme relations autour pour voir un peu » (E16).

La constitution du réseau personnel local est donc facilitée quand le nouvel arrivant a quelques référents sur place. Cependant, le réseautage affectif, c'est à dire l'activation du réseau préexistant, est largement insuffisant. C'est pourquoi un réseutage plus instrumental est mis en place par les enquêtés.

\subsubsection{La nécessité de recourir à un réseautage instrumental pour la constitution du réseau personnel local}

Les éléments de discours concernant la constitution du réseau local font ressortir un certain nombre de pratiques plus formelles que l'activation du réseau préexistant. Il apparaît, donc, qu'à ce stade, le recours à un réseautage instrumental est nécessaire. Ainsi, le rôle du maire et des « élites » locales est 
reconnu comme primordial pour favoriser la constitution du réseau local, comme en témoigne le verbatim suivant : « Je parlais des maires tout à l'heure, c'est des caisses de résonance importantes, en fait, y compris, même s'ils n'ont rien à voir ni avec la commercialisation, ni avec la technique, tout ça. En milieu rural, c'est quand même là que se fait l'intérêt pour l'activité et donc, la réputation aussi. En gros, est-ce que c'est un rigolo ou est-ce que c'est quelqu'un de sérieux ? C'est quand même principalement par les structures communales que ça va passer» (E3). Si la présentation au maire lors de l'arrivée est identifiée comme un minimum, d'autres pratiques sont nécessaires pour être connu (et donc être susceptible d'être contacté ou écouté par de futurs alter) : implication dans la vie de la commune, participation aux réunions et manifestations locales, présentation aux habitants, inauguration de l'entreprise, etc.

Une autre démarche plus formelle de constitution d'un réseau local est le recours aux institutions locales pas seulement à des fins de conseils mais à des fins de réseau. Dès la préparation du projet ou par la suite, certains se rapprochent de structures d'accompagnement locales dans l'optique d'être mis en relation et de se faire connaître plutôt que pour obtenir des conseils et informations. Ainsi, 26 des 56 institutions ou élus évoqués par les enquêtés ont permis l'obtention de ressources relationnelles (mise en relation ou réputation) ou ont été contactés dans cet objectif là. C'est par exemple ce dont se rendent compte ces deux associés dans une société de création de sites Internet, qui sont, au moment de l'entretien, proches du dépôt de bilan : «En fait, on se rend compte a-priori trop tard que le fait d'aller souvent à la chambre d'Industrie fait que l'on commence à vous connaître, donc les autres vous connaissent. [...] Mais ça, aucun créateur ne peut le savoir. C'est après qu'on s'en rend compte. Je ne connais pas un créateur dans les gens que je connais qui ont créé des entreprises en Ardèche qui y aient pensé au moment où ils ont créé. Voilà. Ils sont allés chercher des conseils mais ils ne sont pas allés chercher des gens. Ils ne sont pas allés chercher un tissu de relations, on va dire. Ben, c'est ce qu'il faudrait. »(E9)

De plus, un certain nombre d'enquêtés ont utilisé, au début, des pratiques telles que le publipostage, la publicité ou la participation à des foires pour se faire connaître et favoriser un bouche à oreille positif. C'est par exemple le cas de ce créateur de bijoux en bois précieux : «Si j'ai surtout fait tous ces types de marchés, c'était aussi pour me faire connaitre dans le coin [...] Donc, le but, ça a été vraiment de faire savoir que j'existais, que je créais des produits et que je voulais en vivre. » (E18)

Enfin, certains enquêtés ont adopté une stratégie d'implantation en deux phases (salariat puis création). Pour ce créateur d'une agence de communication sui a créé 5 ans après s'être installé en Ardèche en tant que salarié d'une agence de communication locale, cette stratégie a été un atout majeur dans la constitution d'un réseau local : «Je ne pouvais pas imaginer un instant en 2000 de pouvoir faire ce que je fais aujourd'hui, quoi. C'est impossible, surtout dans les régions comme celle-ci. Il faut vraiment être connu pour être bien apprécié.» (E14) Bien que ce type de stratégie soulève la question de la clause de non-concurrence, il convient de passer du salariat à la création de son entreprise non seulement dans la même zone géographique mais également, si possible, dans le même domaine. Par exemple, l'expérience est plus nuancée pour ce couple créateur d'une société de création de sites Internet : «On était déjà dans l'Ardèche mais par contre, professionnellement, on n'avait pas de réseau. C'était un réseau personnel. [...] On aurait eu un employeur dans la même...mais même dans la même catégorie de services, on va dire, avant, le réseau serait déjà fait. Voilà. C'est parce qu'on ne connaissait pas les gens et qu'il faut tout inventer. » (E9)

Le réseautage instrumental apparaît donc particulièrement pertinent au moment de la constitution du réseau personnel local. Par la suite, quand un premier noyau de réseau local est constitué, les pratiques deviennent plus informelles et on peut parler de réseautage affectif. 


\subsubsection{Le passage à un réseautage plus affectif pour l'entretien et le développement du réseau personnel local}

Dès qu'un premier noyau de réseau personnel local est constitué, donc dès que le contexte fonctionnel « local» devient plus familier, l'entretien et le développement du réseau personnel local repose sur un réseautage plus affectif.

Les enquêtés qui ont le sentiment d'être bien intégrés développent assez rapidement leur réseau personnel local à partir de liens essentiellement amicaux. C'est, par exemple, le cas de ce couple créateur d'une structure d'hébergement pour handicapés mentaux et touristes "C'est le fait d'habiter là et comme les relations sont très bonnes dans le village, on nous dit : il y a ceci, il y a telle personne là, telle personne là. On est présentés aux uns, aux autres et puis, le relationnel s'est fait comme çà, quoi. En quelques mois, on a pu se créer comme ça un petit tissu de partenaires sympas ». (E17). Cependant, ce réseautage affectif n'est possible que parce qu'au préalable, ils ont utilisé un réseautage plus instrumental (présentation au maire, présentation aux habitants par l'ancien propriétaire, recours à des ressources locales pour les travaux, inauguration, contact auprès des institutions locales d'accompagnement, etc.).

L'activation du premier noyau de réseau personnel local constitue également une forme de réseautage affectif. C'est par exemple le cas de ce créateur d'une agence de communication : " En fait, mon meilleur support de communication, ce sont mes clients. C'est eux qui en parlent le mieux. [...] Je pense que, vraiment, c'est la meilleure publicité. Et c'est eux qui démarchent pour moi, directement et inconsciemment, sans le savoir. Tu devrais regarder un tel, essayer de travailler avec... »(E14). D'ailleurs, ces nouveaux alter locaux entrés dans le réseau par "cooptation », c'est à dire sur les recommandations d'un autre alter local, ont une forme de confiance en lui particulière. Il s'agit d'une confiance en la qualité de l'information fournie par la tierce personne évoquée par Hite (2005) : « Il y a même des gens avec qui j'ai travaillé alors que physiquement je ne les connais pas parce que, eux, ont eu mon adresse par quelqu'un d'autre et ils ont confiance. Alors on ne se rencontre pas. C'est extraordinaire quand même comme truc ! C'est fabuleux d'arriver à un niveau comme ça ! (E14)

La constitution d'un réseau local s'appuie, donc, sur un réseautage davantage instrumental (recours au élus et institutions locales, prospection, publicité, participation à des manifestations locales, stratégie d'implantation en deux temps) qu'affectif (activation du réseau préexistant local). Cela permet de se faire connaitre et de déclencher un bouche à oreille positif. Par la suite, quand il s'agit surtout d'entretenir et de développer un premier noyau de réseau local, le réseautage devient plus affectif. En parallèle, les enquêtés créent, entretiennent et développent des relations plus éloignés géographiques. L'objet de la section suivante est de présenter les résultats concernant ce réseautage non-local.

\subsection{Le réseautage non-local : surtout affectif}

Lorsque le contexte fonctionnel est familier (quadrant en haut à droite de la typologie), le rôle joué par le réseau préexistant est très important et le réseautage est donc affectif. Or ces liens doivent être entretenus malgré la distance géographique. Lorsque le contexte fonctionnel est nouveau (quadrant en bas à droite de la typologie), le réseautage peut être affectif (activation du réseau social préexistant) ou instrumental (mais ici, les pratiques sont différentes de celles utilisées pour le réseautage local)

\subsubsection{Contexte fonctionnel familier et réseautage affectif}

Tout d'abord, lorsque le contexte fonctionnel est familier (quadrant en haut à droite de la typologie), la pratique dominante est bien entendu l'activation du réseau professionnel préexistant. C'est par exemple le cas de ce formateur : " j'ai eu différents réseaux dans différents réseaux professionnels dans ma vie antérieure, antérieure à mon installation en Ardèche. [...] Effectivement, j'ai retiré un petit peu les fils » 
(E16). Souvent, ce réseau professionnel préexistant se situe essentiellement dans le milieu d'origine. C'est par exemple le cas de ce créateur d'une entreprise d'étude et de fabrication de produits électroniques : «((Ville d'Origine)), ça correspond à une région que je connais bien. Donc, plutôt que de faire de la prospection, je préfère utiliser le tissu relationnel que j'ai eu pendant de nombreuses années. ». ( E8). Quand le contexte fonctionnel de l'entreprise est familier à l'enquêté, le réseautage non-local correspond donc au cadre général repéré dans la littérature : le réseautage est d'abord affectif.

Le réseautage affectif est très fréquent pour développer le réseau personnel non-local. Ainsi, le bouche à oreille et la cooptation est même parfois le seul moyen de trouver de nouveaux clients. En effet, les enquêtés ayant une clientèle très dispersée sont souvent sur des métiers où le bouche à oreille et la cooptation sont très importants. C'est par exemple le cas de ce créateur d'une entreprise de conseils en organisation : «Dans le métier que je fais, en fait, vous avez beau faire des mailings et tout ça, les gens se parlent par ouïe-dire et vous commandent des activités. Donc, vous avez beau faire des mailings, etc., et tout, en conseil, ça ne sert strictement à rien, ni plus ni moins, ça ne sert à rien. Donc, j'ai déjà essayé une multitude de fois donc je sais. » (E7)

Cependant, se pose le problème de l'entretien des liens malgré la distance géographique. Les enquêtés tentent de pallier le manque de proximité géographique par le recours à des techniques de communication à distance (téléphone, mail, chat, etc.). En effet, cela permet de partager ou de co-produire des connaissances codifiées mais aussi tacites " grâce à l'évolution technologique de l'informatique qui laisse de plus en plus de place à la communication informelle ou visuelle (association de l'image, de l'écrit de la voix) ou à une communication écrite proche de la communication orale (e-mails, forums, chats...) » (Rallet et Torre, 2004, p. 34). Par exemple, ce créateur de site Internet décrit la façon dont il interagit avec ses clients et sous-traitants : «On se contacte par e-mail, téléphone, forum et 2 fois par an, on se rencontre dans des meetings et salons informatiques. Il y a deux ans, on a créé un forum pour échanger. » (E1)

Un autre moyen, souvent complémentaire au recours aux TIC, d'entretenir le réseau non-local est d'effectuer des déplacements. Cela permet de créer une proximité géographique temporaire. En effet, souvent, la contrainte de proximité géographique dans une relation n'est pas permanente. Elle ne porte que sur certains moments de l'interaction. (Rallet et Torre, 2004) Par exemple, cette créatrice d'une entreprise de conseil en gestion de patrimoine et agence immobilière a un réseau (clients, confrères et agents immobiliers) très dispersé, ce qui l'oblige à passer un tiers de son temps en déplacement :« On va dire que j'organise une fois par mois mes déplacements qui me prennent 10 jours à peu près. [...] C'est ma force de prendre ma voiture et de faire $400 \mathrm{~km}$. Ça ne me dérange pas du tout mais alors là. C'est à dire, moi, je suis vraiment nomade, quoi. » (E12)

Enfin, il convient de souligner que l'entretien de relations à distance est facilité quand une proximité autre que géographique existe ou se crée entre alter et ego. Ce type de proximité nommée « de similitude » par Rallet (2004) ou «cognitive »par Julien (2005) est liée au partage de mêmes connaissances de base et d'expertise ou d'un même système de représentions. Ainsi, alors que la plupart de ses clients refusent un traitement à distance, ce dirigeant d'une entreprise informatique a noué une relation particulière avec l'un d'entre eux : «ça fait quand même depuis 3 ans qu'on travaille ensemble, donc maintenant il y a une relation de confiance, donc il accepte que je travaille à distance sans craintes. Ça fait partie des gens qui ont passé ce cap et qui font confiance pour qu'on puisse travailler à distance. » (E11)

Le réseautage non-local quand le contexte fonctionnel est (ou est devenu) familier semble donc être essentiellement affectif. C'est également souvent le cas quand le contexte fonctionnel est nouveau.

\subsubsection{Contexte fonctionnel nouveau et réseautage affectif}

Pour ceux dont le contexte fonctionnel est moins familier, le réseau préexistant joue également un rôle important mais dans ce cas, ce n'est pas le réseau professionnel mais social qui est activé. Il s'agit de 
relations personnelles (ami, famille, connaissance), qui peuvent se situer dans le milieu d'origine mais aussi ailleurs, qui, à un moment donné, vont jouer un rôle dans la création de l'entreprise. C'est le cas, par exemple, de ce créateur d'une activité agritouristique : «Comme il se trouve que pour monter mon projet, j'ai eu besoin de créer une société SCI immobilière, là, par contre, j'ai contacté beaucoup de gens, activé tout un réseau personnel. [...] c'est vraiment des relations personnelles. » (E3).

Dans certains cas, le réseau qui est activé est le réseau local précédemment constitué. Par exemple, cette traductrice a créé de nouveaux liens géographiquement éloignés à partir de contacts locaux : «Donc, A [Traductrice en Ardèche], elle m'avait déjà refilé tels ou tels travaux à faire. Et puis, donc j'ai connu à travers les B ((club de conversation anglaise)) encore d'autres amies qui m'ont introduite auprès de l'une de leur agence, qui eux m'ont trouvé du travail aussi, assez vite fait. » (E10).

Cependant, le réseautage affectif semble insuffisant quand le contexte fonctionnel est nouveau. C'est pourquoi, un réseautage plus instrumental est également utilisé pour constituer un réseau personnel non-local.

\subsubsection{Contexte fonctionnel nouveau et réseautage instrumental : le recours à Internet plutôt qu'à la publicité, au démarchage ou à la participations à des foires/salons}

Une pratique de réseautage instrumental très répandue pour se constituer un réseau non-local est le recours à Internet. Internet peut être utilisé pour rechercher et entrer en contact avec de nouvelles relations comme des fournisseurs, des distributeurs ou des clients potentiels (réponse à des appels d'offres par exemple). Internet est, aussi, utilisé pour se faire connaître à un niveau national ou international. La démarche de constitution d'un réseau non-local de ce créateur de sites Internet est particulièrement illustrative de cette pratique : «J'ai réalisé tout un marketing internet. J'ai participé à des concours de référencement, à des concours internationaux. Et ça m'a permis de me faire reconnaître dans le secteur. A partir de là, j'ai commencé à travailler en sous-traitance pour d'autres personnes du secteur.» (E1)

Pour se faire connaître dans son secteur d'activité mais aussi échanger avec d'autres acteurs de ce secteur, les sites d'indépendants sont un outil efficace, en particulier pour les activités intellectuelles. C'est par exemple, un outil utilisé par ce dirigeant d'une société de prestations informatiques : « Depuis que j'ai commencé, je me suis inscrit sur pas mal de sites d'indépendants. Donc, ça me permet en fait de connaître des nouvelles sociétés qui cherchent. Et puis, par-là, d'avoir des contacts. Ça a déjà débouché. Je suis déjà rentré en contact avec des sociétés de services informatiques via des sites d'indépendants.» (E11)

Enfin, le site Internet de l'entreprise est un outil majeur dans la création de liens nouveaux géographiquement éloignés. Ainsi, 12 enquêtés ont un site Internet (pour 3 d'entre eux, il s'agit d'un site marchand) et 3 envisagent d'en créer un.

En revanche, des pratiques telles que le démarchage commercial, la publicité ou la participation à des salons sont trés peu utilisées alors qu'elles le sont assez fréquemment pour la constitution du réseau local. La publicité est peu utilisée pour se faire connaître à un niveau national ou international car trop coûteuse. En plus de leur coût, les pratiques telles que la participation à des salons ou le démarchage commercial sont également très preneuses en temps, comme le soulignent ce créateur d'une entreprise d'étude et fabrication de produits électronique : "C'est vrai qu'aujourd'hui je ne vais pas à des salons, des choses comme ça. C'est vrai que d'aller à un salon, ça permet de rencontrer des gens, de discuter aussi, hein. Mais bon, on ne peut pas tout faire non plus. Tout ça, c'est pas facile. » (E8)

Le réseautage non-local est donc avant tout affectif (activation du réseau, social et professionnel, préexistant, activation du réseau personnel local, cooptation/bouche à oreille). Le réseautage instrumental existe également dans un contexte non-local mais il est différent du réseautage instrumental 
local car il s'appuie essentiellement sur le recours à Internet et peu sur la publicité, le démarchage ou la participation à des salons/foires.

\section{Conclusion}

Le cas des entrepreneurs néo-ruraux constitue un angle d'entrée nouveau dans la problématique du réseautage. En effet, l'étude exploratoire conduite auprès de 20 cas implantés dans les Monts et Montagnes d'Ardèche permet de proposer une typologie des pratiques de réseautage. Ces dernières sont positionnées sur un plan à deux axes : la familiarité de l'entrepreneur avec le contexte fonctionnel de son entreprise et le contexte géographique dominant. Or, les pratiques de réseautage local et non-local différent grandement.

Pour constituer un réseau personnel local, les entrepreneurs néo-ruraux mettent en place des pratiques allant au-delà de la simple activation d'un réseau préexistant. En effet, le réseautage affectif est rarement suffisant. En fait, le réseautage instrumental est primordial au début. Ainsi, les élus, « élites » et institutions locales sont un point d'entrée essentiel dans le territoire car ils permettent d'être mis en relation avec des contacts potentiels et créent, en partie, la réputation du nouvel arrivant. Pour se faire connaître, certains enquêtés utilisent également la prospection, la publicité et la participation à des foires locales. Enfin, la stratégie de création en deux temps (migration en tant que salarié puis création) permet, quand l'emploi salarié est occupé dans le même secteur, de se créer un réseau professionnel local avant la création. Par la suite, le réseautage devient plus affectif et le réseau personnel local se développe surtout par bouche à oreille et cooptation.

Contrairement à la constitution du réseau local, la constitution du réseau non-local s'appuie essentiellement sur l'activation d'un réseau social et/ou professionnel préexistant, souvent localisé dans le milieu d'origine. Parfois, le réseau local peut également servir d'appui vers l'extérieur. Donc le réseautage non-local est essentiellement affectif Les méthodes formelles employées pour se faire connaître au niveau local (démarchage, publicité, participation à des salons) sont, ici, peu utilisées. En revanche, le rôle d'Internet est très important pour se faire connaître. De plus, nous avons montré que l'activation, la création et l'entretien de relations géographiquement éloignées impliquent l'utilisation des TIC et/ou des déplacements permettant une proximité géographique temporaire. Cependant, l'existence d'une proximité cognitive entre alter et ego, proximité qui peut être liée à une proximité géographique passée (par exemple, dans le milieu d'origine) ou à l'existence d'affinités personnelles, facilite grandement, voire est indispensable dans ce type de relations à distance.

Ces résultats présentent un certain nombre d'implications quant à l'accompagnement des entrepreneurs néo-ruraux. Tout d'abord, en soulignant les freins à la constitution d'un réseau local liés au caractère de nouvel arrivant, nos résultats justifient l'attitude de nombreuses structures d'accompagnement qui cherchent à faciliter l'insertion locale de l'entrepreneur néo-rural. Pour cela, un certain nombre d'outils existent : sessions de présentation du territoire, parrainage par (ou mise en relation avec) des dirigeants d'entreprises locales, création de clubs, organisation de réunions d'échanges, sensibilisation des élus et de la population locale à l'accueil de nouveaux arrivants, etc. Cependant, ces résultats invitent également les structures d'accompagnement à encourager l'entrepreneur néo-rural à ne pas se focaliser uniquement sur la constitution du réseau personnel local. En effet, compte tenu des handicaps du milieu rural, la constitution d'un réseau personnel non-local est un impératif. Or, ceci implique des pratiques de réseautage différentes de celles utilisées pour la constitution du réseau local. Une attention toute particulière doit être porté sur l'activation du réseau social et/ou professionnel préexistant, notamment dans le milieu d'origine, sur le recours à Internet et sur l'utilisation des TIC et de déplacements.

Toutefois, notre étude présente certaines limites. Tout d'abord, notre observation se base sur un échantillon relativement limité (20 cas) et s'appuie sur des entretiens semi-directifs rétrospectifs. Or, ce mode de recueil présente un biais d'oubli et de rationalisation a posteriori. De plus, les entreprises 
étudiées sont jeunes et en phase de démarrage. Aussi, la réussite de ces dernières est difficile à évaluer. Par conséquent, de futures études pourraient être conduites de façon longitudinale afin de confronter pratiques de réseautage utilisées et réussite de l'entreprise. A partir de là, des « meilleures pratiques » pour chaque logique de réseautage pourraient être identifiées.

\section{Notes}

1. Traduction de «As an alternative to under- and oversocialized models of entrepreneurship, we propose a perspective that views entrepreneurship as embedded in networks of continuing social relations. » (Aldrich et Zimmer, 1985,p. 8)

2. Ego et alter sont des termes issus de l'analyse structurale des réseaux sociaux. Dans le texte, ego désigne l'entrepreneur, alter les membres du réseau

3. Beaucoup d'auteurs dans le courant de l'entrepreneur « encastré » mobilisent le concept de capital social. Parmi les deux grandes approches du capital social (Adler et Kwon, 2002), comme bien collectif et comme bien individuel, c'est cette dernière qui a été retenue par la plupart des auteurs en entrepreneuriat puisqu'elle s'intéresse à la façon dont un acteur individuel, en l'occurrence l'entrepreneur, « investit » dans certaines relations et comment ces relations peuvent l'aider. Cependant, malgré son utilisation grandissante en sciences de gestion, il semble qu'un consensus sur la définition du concept de capital social comme bien individuel soit impossible. Ainsi, Chollet (2004) identifie trois éléments présents de façon récurrente dans les définitions du capital social comme bien individuel : le portefeuille de relations personnelles de l'individu (le réseau), les ressources qui sont accessibles par ce réseau et la performance individuelle associée à ces ressources. Quatre configurations sont alors identifiées. Selon les auteurs, le capital peut être assimilé aux ressources accessibles par le réseau personnel (Bourdieu, 1980, Lin, 2001), au bénéfice tiré de la place de l'individu dans la structure du réseau, donc au réseau lui-même (Burt, 1995), aux dispositions favorables ou aux obligations ressenties dans le réseau (Adler et Kwon, 2002) ou englober à la fois le réseau et les ressources qui y sont inscrites (Nahapiet et Ghoshal, 1998, Portes, 1998). Compte-tenu de la polysémie du concept même de capital social, et donc de la diversité des usages qui en sont fait en entrepreneuriat, nous considérons que le capital social est en fait une métaphore et nous préférons parler le texte de « ressources », de « réseau personnel », de « dispositions favorables/normes » ou de « processus de mobilisation de ressources par le réseau personnel » bien que certains auteurs utilisent parfois le terme « capital social » pour qualifier ces éléments

4. Le terme « réseautage » est une traduction littérale de l'expression "networking », utilisée dans les travaux anglo-saxons. S'il est peu utilisé en France, son utilisation est courante au Québec. Nous l'utilisons comme synonyme de « constitution et mobilisation du réseau personnel ».

5. Traduction de «Mobilizing resources to pursue opportunities require entrepreneurial contacts, knowledge, and confidence. Mobilizing resources also involves asking others to raise money, labor, and effort for a venture with an uncertain future. Entrepreneurship is thus inherently a networking activity. » (Dubini et Aldrich, 1991, p.306)

6. Traduction de «The strongest evidence supporting the importance of social networks is that most entrepreneurs start new firms at home »; at home in familiar geographical contexts - same community and political juridictions - as well as industry contexts » (Reynolds, 1991, p. 64)

7. Sondage réalisé en avril 2007 par l'institut BVA auprès de 457 personnes formant un échantillon représentatif de citadins. Résultats consultables sur le site www.projetsencampagne.com

8. Traduction de «process, taking place over time, a dynamic relationship that involves shifting latent contact to manifest ties, transferring relationships to the entrepreneurial situation, identifying entrepreneneurial requirements and locating an individual within the network who can help with the actual need. » (Jack, 2005, p. 1251)

9. Traduction de « These shared normative and performance values were used as a hiring criterion for employees, but also as a way of validating the choice of operating parteners outside the firm. It was seen as basis for friendship relationships - for the developing of strong from weak ties » (Drakopoulou Dodd et al., p. 134)

10. SE : Salarié Entrepreneur d'une entreprise coopérative permettant l'hébergement, le test et le développement d'activités économiques.

\section{Bibliographie}

ADLER P-S., KWON S-W (2002), «Social capital: Prospects for a new concept », Academy of Management. The Academy of Management Review, 27(1)

ALDRICH H., ROSEN B., WOODWARD W-J. (1987), « The impact of social networks on business foundings and profit: an longitudinal study », dans NC CHURCHILL, JA HORNADAY, BA KIRCHHOFF, OJ KRASNER, KH VESPER (dir.), Frontiers of Entrepreneurship Research, Babson College

ALDRICH H., ZIMMER C. (1985), «Entrepreneurship through Social Networks », dans DL SEXTON, RW SMILOR (dir.), The art and science of entrepreneurship. Ballinger Publishing Company : 3-24 
ANDERSON A. (2000), « The protean entrepreneur : the entrepreneurial process as fitting self and circumstance», Journal of Enterprising Culture, 8(3): 201-234

ANDERSON A., JACK S. (2002), «The articulation of social capital in entrepreneurial networks: a glue or a lubricant? ?, Entrepreneurship and Regional Development, 11: 198-210

ASSOGBA Y., FRÉCHETTE L., DESMARAIS D. (2000), « Le mouvement migratoire des jeunes au Québec. La reconfiguration du réseau social, un repère pour étudier le processus d'intégration », Nouvelles Pratiques Sociales, 13(2): $65-78$

BARON R-A., MARKMAN G-D. (2000), «Beyond social capital: How social skills can enhance entrepreneurs' success », The Academy of Management Executive 14(1): 106

BARON RA., MARKMAN G-D. (2003), «Beyond social capital: The role of entrepreneurs' social competence in their financial success », Journal of Business Venturing, 18(1): 41

BERGER-DOUCE S. (2006), «L'accompagnement des éco-entrepreneurs: une étude exploratoire », 8ème Congrès International Francophone en Entrepreneuriat et PME: Fribourg, Suisse

BIDART C., FRIBOURG B. (2004), «Qui sont mes proches?" Proximités spatiales, proximités sociales dans les évolutions des réseaux relationnels de jeunes entrant dans l'âge adulte ", Quatrièmes journées de la proximité: Marseille, 17-18 juin

BONCLER J., HLADY-RISPAL M. (2002), Caractérisation de l'entrepreneuriat en économie solidaire. Les éditions de l'ADREG (http://www.adreg.net)

BOURDIEU P. (1980), «Le capital social, notes provisoires », Actes de la recherche en sciences sociales, 31: 2-3

BOWEY JL., EASTON G. (2007), «Entrepreneurial social capital unplugged: An activity-based analysis », International Small Business Journal 25(3): 273-306

BURT RS. (1995), «Le capital social, les trous structuraux et l'entrepreneur », Revue Française de Sociologie, 36: 599-628

BUTLER JE., HANSEN EL. (1991), « Network evolution, entrepreneurial success, and regional development », Entrepreneurship and Regional Development, 3(1): 1-16

CARRIER C., RAYMOND L., ELTAIEF A. (2002), «Le cyberentrepreneuriat: une étude exploratoire », Revue internationale PME, 15(3-4): 139-162

CARSON D., CROMIE S., MCGOWAN P., HILL J. (1995), Marketing and Entrepreneurship in SMEs, Prentice-Hall: Englewood Cliffs, NJ

CHELL E., BAINES S. (2000), « Networking, entrepreneurship and microbusiness behaviour », Entrepreneurship and Regional Development, 12: 195-215

CHEVALIER P. (2003), «Qualité de vie et logique économique dans les espaces de faible densité », Revue d'Economie Méridionale, 51(201-202): 149-153

CHOLLET B. (2004), "Théories et mesures du capital social dans la recherche en management », 17èmes journées nationales des IAE: Lyon, 13-14 septembre

COLLECTIF VILLE CAMPAGNE (2003), Bilan des actions en faveur de l'accueil de nouveaux actifs en milieu rural. DATAR:

DRAKOPOULOU DODD S., JACK S., ANDERSON A (2006), «The Mechanisms and Processes of Entrepreneurial Networks: Continuity and Change », dans DD JOHAN WIKLUND, JEROME A. KATZ and DEAN A. SHEPHERD (dir.), Entrepreneurship: Frameworks And Empirical Investigations From Forthcoming Leaders Of European Research, Vol. 9. Advances in Entrepreneurship, Firm Emergence and Growth JAI Press: 
DUBINI P., ALDRICH H. (1991), «Personal and Extended Networks Are Central to the Entrepreneurial Process », Journal of Business Venturing, 6(5): 305

EMIN S. (2003), L'intention de créer une entreprise des chercheurs publics: le cas français, Thèse de Doctorat en Sciences de Gestion: Université Pierre Mendès-France Grenoble

GRANOVETTER M. (1985), «Economic Action and Social Structure: A theory of Embeddedness », American Journal of Sociology, 91(3): 481-510

GROSSETTI M. (2001), «Les effets de proximité spatiale dans les relations entre organisation: une question d'encastrements », Espaces et Sociétés, (101-102): 203-219

HANSEN EL. (1995), «Entrepreneurial networks and new organisation growth », Entrepreneurship Theory and Practice, 19(4): 7-20

HILL J., MCGOWAN P., DRUMMOND P. (1999), « The development and application of a qualitative approach to researching the marketing networks of small firm entrepreneurs », Qualitative Market Research, 2(2): 71-81

HITE JM. (2005), «Evolutionary Processes and Paths of Relationally Embedded Network Ties in Emerging Entrepreneurial Firms », Entrepreneurship Theory and Practice, 29(1): 113

HOANG H., ANTONCIC B. (2003), « Network-based research in entrepreneurship: A critical review », Journal of Business Venturing, 18(2): 165

JACK SL. (2005), «The role, use and activation of strong and weak network ties: a qualitative analysis », The Journal of Management Studies, 42(6): 1233-1258

JOHANNISSON B. (1986), «Network strategies: management technology for entrepreneurship and change », International Small Business Journal, 5(1): 19-30

JOHANNISSON B. (1987), «Beyond process and structure: social exchange networks », International Studies of Management \& Organization 17(1): 3-23

JOHANNISSON B., MONSTED M. (1997), « Contextualizing entrepreneurial networking », International Studies of Management \& Organization 27(3): 109-136

JOYAL A. (2000), «PME rurale: une revue de la documentation », L'Encyclopédie de l'Agora:

JULIEN P.A. (2005), Entrepreneuriat régional et économie de la connaissance: une métaphore des romans policiers, Les Presses Universitaires du Québec

JULIEN P-A, LACHANCE R. (2006), «Réseaux personnels, d'affaires et informationnels: une dynamique complexe », 8ème Congrès International Francophone en Entrepreneuriat et PME: Fribourg, Suisse, 25-27 octobre

KALANTARIDIS C, BIKA Z. (2006), «In-migrant entrepreneurship in rural England: beyond local embeddedness », Entrepreneurship and Regional Development, 18: 109-131

LEVY-TADJINE T. (2004), L'entrepreneuriat immigré et son accompagnement en France, Thèse de Doctorat en Sciences de Gestion: Institut d'Administration des Entreprises. Université du Sud Toulon-Var

LEVY-TADJINE T., PATUREL R. (2006), « Modéliser et singulariser le phénomène entrepreneurial », 8ème Congrès International Francophone en Entrepreneuriat: Fribourg, Suisse, 25-27 octobre

LIN N. (2001), «Building a Network Theory of Social Capital », dans N LIN, RG COOK, RS BURT (dir.), Social Capital - Theory and Research. Aldine de Gruyter: 3-29 
LUX S., FERRIS GR. (2006), «Developing and utilizing efficient ties: The role of ego capabilities in entrepreneurial networks ", Workshop in strategic entrepreneurship: the role of networking: Vrije University Amsterdam, 3-4 juillet

MINGUET G., MOREAU R. (2006), «La construction sociale des réseaux de soutien des entreprises émergentes », Colloque International: Création d'entreprises et territoires: Tamanrasset, 2-3 décembre

MITCHELL JC. (1973), «Networks, norms and institutions », dans J Boissevain, JC Mitchell (dir.), Network Analysis. Studies in Human Interaction. The Hague: 2-35

MOREL B, REDOR P. (2006), «Enquêtes annuelles de recensement 2004 et 2005: La croissance démographique s'étend toujours plus loin des villes », INSEE Première, (1058)

NAHAPIET J., GHOSHAL S (1998), «Social Capital, Intellectual Capital and the Organizational Advantage », Academy of management review, 23(2): 242-266

NEGRO Y. (1995), «L'héritier, le natif et le "néo": Trois profils de créateurs d'entreprises non agricoles en milieu rural », Revue d'Economie Méridionale, 43(172): 39-57

O'DONNELL A., GIMORE A, GUMMINS D., CARSON D. (2001), « The network construct in entrepreneurship research: a review and critique », Management Decision, 39(9): 749-760

OBSERVATOIRE DES TERRITOIRES (2005), Dynamique et diversité des territoires français. La Documentation française: Paris

PORTES A. (1998), «Social Capital: Its Origins and Applications in Modern Sociology », Annual Review of Sociology, 24(1): 1-24

REYNOLDS PD. (1991), «Sociology and Entrepreneurship: Concepts and Contributions », Entrepreneurship: Theory \& Practice, 16(2): 47-70

ROUSSEL V., BÉGON M. (2005), «Les nouveaux venus et leur rapport au territoire: Etude de cas dans le Massif Central », Revue de l'Economie Méridionale, 53(212): 463-481

SCHILDT HA., ZAHRA SA., SILLANPAA A. (2006), « Scholarly Communities in Entrepreneurship Research: A Co-Citation Analysis », Entrepreneurship Theory and Practice, 30(3): 399-415

SHIELDS JF. (2005), «Does rural location matter? The significance of a rural setting for small business », Journal of Developmental Entrepreneurship 10(1): 49-63

STARR JA. ET MACMILLAN IC. (1990), « Resource cooptation via social contracting: ressource acquisition strategies for new ventures », Strategic Management Journal, 11: 79-92

SZARKA J. (1990), « Networking and Small Firms », International Small Business Journal, 8(2): 10-22

VERSTRAETE T., SAPORTA B. (2006), Création d'entreprise et Entrepreneuriat, Les Editions de l'ADREG (http://www.adreg.net)

VISSA B., ANAND N. (2006), «Entrepreneurs' networking styles: impact on tie formation and dissolution during venture emergence », Academy of Management Best Conference Paper

WATSON J. (2007), « Modeling the relationship between networking and firm performance », Journal of Business Venturing, 22(6): 852-874 